\title{
Photoinduced fluidity in chalcogenide glasses at low and high intensities: A model accounting for photon efficiency
}

\author{
Yann Gueguen, ${ }^{1}$ Jean Christophe Sangleboeuf, ${ }^{1, *}$ Vincent Keryvin,,${ }^{1,2}$ Eric Lépine, ${ }^{3,4}$ Zhiong Yang, ${ }^{3}$ Tanguy Rouxel, ${ }^{1}$ \\ Céline Point, ${ }^{3,4}$ Bruno Bureau, ${ }^{4}$ Xiang-Hua Zhang, ${ }^{4}$ and Pierre Lucas ${ }^{3}$ \\ ${ }^{1}$ LARMAUR Equipe Verres ERL CNRS 6274, Université de Rennes 1, Campus de Beaulieu, 35042 Rennes Cedex, France \\ ${ }^{2}$ LIMATB EA 4250, Université de Bretagne Sud, Rue de Saint Maudé, 56321 Lorient Cedex, France \\ ${ }^{3}$ Department of Materials Science and Engineering, University of Arizona, 4715 E. Fort Lowell Road, Tucson, Arizona 85712, USA \\ ${ }^{4}$ Equipe Verres et Céramiques, UMR-CNRS 6226 Sciences Chimiques de Rennes, Université de Rennes 1, Campus de Beaulieu, \\ 35042 Rennes Cedex, France
}

(Received 30 April 2010; revised manuscript received 28 September 2010; published 18 October 2010)

\begin{abstract}
Detailed measurements of photoinduced fluidity in Ge-Se glasses were performed using a novel shear relaxation test in torsion mode. It is shown that photofluidity is significant even at a very low intensity and that there is no apparent threshold for activating the photostructural processes. Instead, the mechanism of photofluidity is described as a cumulative process involving photoinduced motions of every atom within the irradiated volume. Based on this assumption, a model is proposed, which is shown to accurately predict the power and wavelength dependence of photofluidity using a single fitting parameter $n$. The factor $n$ represents the photon efficiency for inducing an atomic motion. Photofluidity experiments performed on glass fibers of various mean coordination number indicate that the process is rapidly reduced in overconstrained glasses. The values of $n$ obtained for these glasses correlate remarkably well with the mean coordination dependence of other photostructural changes (photodarkening, photoexpansion). This indicates that the model is physically sound. Moreover, the model is shown to quantitatively describe photofluidity data from other glass systems from literature, therefore suggesting that it could be universally applied to all chalcogenide glasses.
\end{abstract}

DOI: 10.1103/PhysRevB.82.134114

PACS number(s): 83.80.Ab, 66.20.Ej, 42.70.Ce

\section{INTRODUCTION}

Chalcogenide glasses exhibit a wide range of photoinduced phenomena, from photodarkening to photocrystallization (see Refs. 1 or 2 for an exhaustive list). Most of the existing models ${ }^{3-6}$ interpret the photoinduced effect through the creation of electron-hole pairs as the result of photoexcitation of chalcogenide lone pairs located at the top of the valence band. Photoexcitation can produce metastable local configuration defects, such as valence alteration pairs (VAP). ${ }^{7}$ These photoinduced defects, localized in band tail states, alter the optical band gap. One of the most spectacular photoinduced effects is the photoinduced fluidity phenomenon (i.e., the decrease of viscosity with irradiation). First evidences of this effect were underlined by Vonwiller ${ }^{8}$ who explained that light has an influence on the distortion rate of selenium fibers. During the 1990s, Hisakuni and Tanaka ${ }^{9}$ were the first to address the critical importance of the light source wavelength (with an energy lower than the Tauc or optical band-gap energy $E_{g}$ ), in order to avoid any significant thermal effects. The authors studied the photoinduced effect at various temperatures on $\mathrm{a} \mathrm{As}_{2} \mathrm{~S}_{3}$ glass and showed that viscosity increases with temperature, therefore clearly demonstrating that photoinduced fluidity is athermal. An equivalent study on pure glassy selenium has also demonstrated this temperature dependence. ${ }^{10}$ Yannopoulos et al. ${ }^{11}$ have recently given a general overview of photoinduced mechanical effects that complements one of Tanaka. ${ }^{12}$ Some quantitative experiences have evidenced the photofluidity ${ }^{8,13,14}$ but did not provide any key to understand this effect. Trunov et al. ${ }^{15}$ have provided interesting experimental data on the topic and a strong demonstration of the athermal nature of photofluid- ity through polarization effects. ${ }^{16}$ Nemilov et al. ${ }^{17}$ have also shown that the viscosity exponentially decreases with light intensity and also depends on the wavelength (see Ref. 11 for a summary in English). This tendency has been interpreted through a decrease in energy barriers for viscous flow. Kastrissios et al. ${ }^{18}$ have also studied the photofluidity through Raman scattering to analyze its structural origin and its temperature dependence. Nevertheless, so far, no model is able to describe quantitatively the viscosity dependence to the irradiation conditions.

The mechanism leading to photoinduced fluidity is not fully understood as yet. Tanaka suggested that an intensity (or irradiance) "threshold" exists for photofluidity ${ }^{12}$ because under low light intensity exposure there is not enough photoinduced events per unit volume to release all the "structural knots" in the network and induce macroscopic fluidity. ${ }^{12,19}$ The density of knots is estimated to be comparable to the density of localized states that corresponds to an intensity threshold close to $\sim 10^{2} \mathrm{~W} / \mathrm{cm}^{2}$ (Ref. 12) in $\mathrm{As}_{2} \mathrm{~S}_{3}$ for a photon energy of $2.0 \mathrm{eV}$. Based on this assumption it is expected that no macroscopic deformation can occur below this threshold. In this study, it is shown that photoinduced fluidity is actually present even at very low light intensity. This observation leads to a reassessment of the mechanism and the origin of photofluidity. We have studied the photoinduced fluidity in the Ge-Se system from low to high intensities at various wavelengths, and we propose a model taking into account the effects of the light intensity, of the wavelength and of the mean coordination of the glassy network.

This paper is divided in five parts: first, we introduce some physical properties of the considered glasses. Then, we demonstrate the existence of photoinduced fluidity under low 
TABLE I. Main physical properties of considered glasses. Elastic shear modulus are measured in the dark. Atoms per millimeter length are given for fibers $300 \mu \mathrm{m}$ in diameter. The viscosities at room temperature are those under thermodynamic equilibrium in darkness.

\begin{tabular}{lcccc}
\hline \hline Glass composition & $\mathrm{GeSe}_{9}$ & $\mathrm{GeSe}_{4}$ & $\mathrm{GeSe}_{3}$ & $\mathrm{Te}_{2} \mathrm{As}_{3} \mathrm{Se}_{5}$ \\
\hline Elastic shear modulus $\mu(\mathrm{GPa})$ & 4.60 & 5.72 & 6.25 & 6.55 \\
Density $\left(\mathrm{g} \mathrm{cm}^{-3}\right)$ & 4.34 & 4.37 & 4.35 & 4.90 \\
Mean coordination number $\langle r\rangle$ & 2.2 & 2.4 & 2.5 & 2.3 \\
Band-gap energy $E_{g}(\mathrm{eV})^{\mathrm{a}, \mathrm{b}}$ & 1.95 & 2.00 & 2.06 & 1.56 \\
Corresponding wavelength $\lambda_{g}(\mathrm{~nm})$ & 636 & 620 & 602 & 795 \\
Reflectance & 0.225 & 0.215 & 0.210 & $/$ \\
Atoms per millimeter length $\left(10^{18}\right)$ & 2.40 & 2.44 & 2.44 & $/$ \\
Viscosity at room temperature $(\mathrm{Pa} \mathrm{s})$ & $10^{19}$ & $10^{25.5}$ & $>10^{30}$ & $>10^{30}$ \\
\hline \hline
\end{tabular}

${ }^{\text {aReference } 24 .}$

${ }^{b}$ Reference 25 .

${ }^{c}$ Reference 26.

light intensity in $\mathrm{GeSe}_{9}$, and we use viscosity measurements at high light intensities on $\mathrm{GeSe}_{9}$ to establish a model taking into account the fluence of the irradiation source and its wavelength, and that it is able to predict the viscosity under low polychromatic light. Then, we use this model to study the mean coordination effect on the sensitivity to photoexcitation. Finally we discuss the implication of the model and we show its good agreement with experimental data in the literature.

\section{MATERIALS}

Four chalcogenide glasses are considered: $\mathrm{GeSe}_{9}, \mathrm{GeSe}_{4}$, $\mathrm{GeSe}_{3}$, and $\mathrm{Te}_{2} \mathrm{As}_{3} \mathrm{Se}_{5}$ (further referred to as "TAS"). The glass production procedure is mainly the following: Starting elements with minimum $99.999 \%$ purity are put into a twochamber silica ampoule which is then vacuum pumped to about $10^{-5}$ mbar. The sealed ampoule is then introduced into a specially design rocking furnace and heated to a temperature of $900{ }^{\circ} \mathrm{C}$ where the melt is highly fluid. The melt is then rocked for $12 \mathrm{~h}$ in order to ensure complete homogeneous mixing of the component elements. After cooling the resulting glass preform is inspected with an infrared camera in order to confirm its homogeneity. Fibers, $300 \mu \mathrm{m}$ (shear relaxation test) and $400 \mu \mathrm{m}$ (creep test) in diameter were then drawn from the homogeneous and well-annealed preforms (see Refs. 20 and 21 for details concerning fibers synthesis).

The elastic shear moduli were calculated from the measurements of ultrasonic wave velocities ${ }^{22,23}$ at $20{ }^{\circ} \mathrm{C}$ and are listed in Table I. The relative error on the shear modulus is $\pm 0.05 \mathrm{GPa}$. The density was measured at $20{ }^{\circ} \mathrm{C}$ by the Archimedean displacement technique using $\mathrm{CCl}_{4}{ }^{22,23}$ The relative error on the density is $\pm 0.5 \%$. Microindentation tests have been performed on fibers using a microindenter Fisherscope HC100, in order to ensure their elastic moduli do not significantly differ from those of bulk glasses.

The average or mean coordination number of chalcogenide glasses is a well-known parameter used to describe many physical behaviors such as fragility and elastic properties. ${ }^{27}$
The mean coordination number is: $\langle r\rangle=\sum_{i} x_{i} r_{i}$, where $x_{i}$ is the atomic fraction of atoms $i$ and $r_{i}$ the coordination number of these atoms.

Considering the molar masses of Ge and Se and the densities of a Ge-Se glasses, we also give the number of atoms per millimeter in a fiber (300 $\mu \mathrm{m}$ in diameter). The viscosities at room temperature are estimated by extrapolating available data around the glass transition temperature $\left(T_{g}\right)$ using Arrhenius laws. ${ }^{28}$

\section{LOW-INTENSITY IRRADIATION}

\section{A. Experiments}

Two chalcogenide glass compositions are considered for low intensity tests: $\mathrm{GeSe}_{9}$ and TAS. Fibers, $300 \mu \mathrm{m}$ (relaxation test) and $400 \mu \mathrm{m}$ (creep test) in diameter, were cut into samples $150 \mathrm{~mm}$ in length. In order to investigate the mechanical behavior of these chalcogenide glasses under low light intensity exposure, we use a low power fluorescent light emitting at energies lower than the Tauc energies of the $\mathrm{Ge}-\mathrm{Se}$ glass. An entire chalcogenide fiber is illuminated and concomitantly submitted to a mechanical test at room temperature $\left(20^{\circ} \mathrm{C}\right)$.

The illuminating light spectrum is presented in Fig. 1 along with the transmission spectrum of $\mathrm{GeSe}_{9}$. The transmission spectrum is measured on a $300-\mu$ m-thick sample. A fluorescent light Phillips MASTER TL-D 36W/840 was used with two bulbs. The distance between the light source and the fibers was $1.2 \mathrm{~m}$. The range of wavelengths capable of inducing consequent bulk effects in $\mathrm{GeSe}_{9}$ glass represents an intensity lower than $165 \mu \mathrm{W} / \mathrm{cm}^{2}$ at this distance (see Fig. 1), the total intensity corresponding to $540 \mu \mathrm{W} / \mathrm{cm}^{2}$. Assuming that the penetration depth of a wavelength is defined as the penetration $(u)$ for which the remaining intensity is $1 / e$ (i.e., for which the absorption is $1 / u$ ), the penetration depth of wavelengths below $\lambda_{g}$, the wavelength corresponding to Tauc energy (see Table I) is lower than $10 \mu \mathrm{m}$. Then, we will assume that only wavelengths over $\lambda_{g}$ must be considered. 


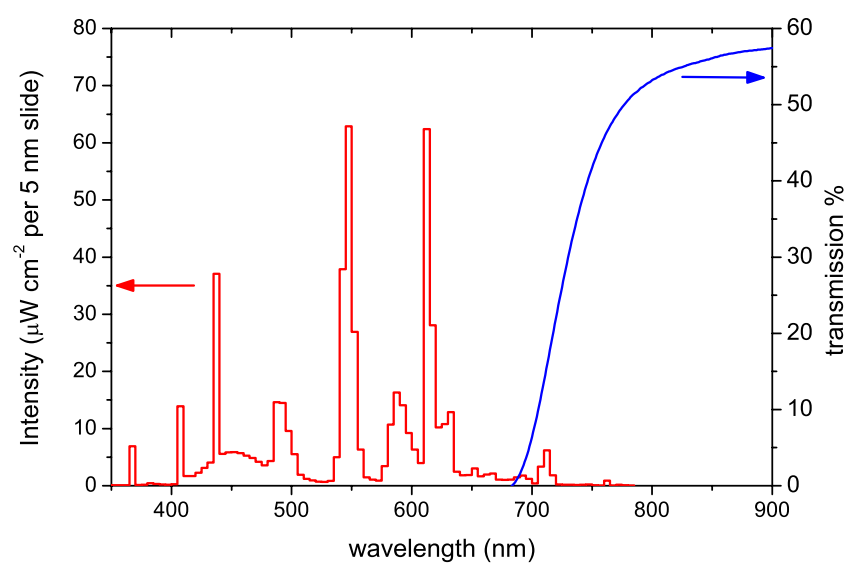

FIG. 1. (Color online) Spectrum of the light emitted at a distance of $1.2 \mathrm{~m}$ from the source (left scale) and transmission spectra (right scale) of $\mathrm{GeSe}_{9}(300-\mu \mathrm{m}$-thick sample). The light spectrum can be read as follows, as an example: a $10 \mathrm{~nm}$ wide peak with an intensity of $3 \mu \mathrm{W} \mathrm{cm}{ }^{-2} / 5 \mathrm{~nm}$ corresponds to a cumulated intensity of $\left.10(\mathrm{~nm}) / 5(\mathrm{~nm}) \times 3(\mu \mathrm{W} \mathrm{cm})^{-2} / 5 \mathrm{~nm}\right)=6 \mu \mathrm{W} \mathrm{cm}{ }^{-2}$ in this $10 \mathrm{~nm}$ range.

\section{Creep test}

Creep tests were performed on as drawn fibers according to the following procedure: the fiber, fixed at one end, supports a carbon tube, articulated on the left (see Fig. 2), and a controlled load is applied on this tube. The stress $(\sigma)$ is calculated taking into account the mass of the carbon tube and that of the fiber holder with a better than $\pm 0.1 \mathrm{MPa}$ accuracy. The applied stress is $15 \mathrm{MPa}$. During creep, the strain $(\epsilon)$, defined as $\Delta L / L$, increases and is a linear function of time in the permanent creep regime. The displacement elongation $\Delta L$ is measured using a laser sensor (Keyence LKG10) with a $10 \mathrm{~nm}$ accuracy. The stationary strain rate, $\dot{\epsilon}$, allows for an estimation of the shear viscosity coefficient $\eta$ $=\sigma /(3 \dot{\epsilon})$.

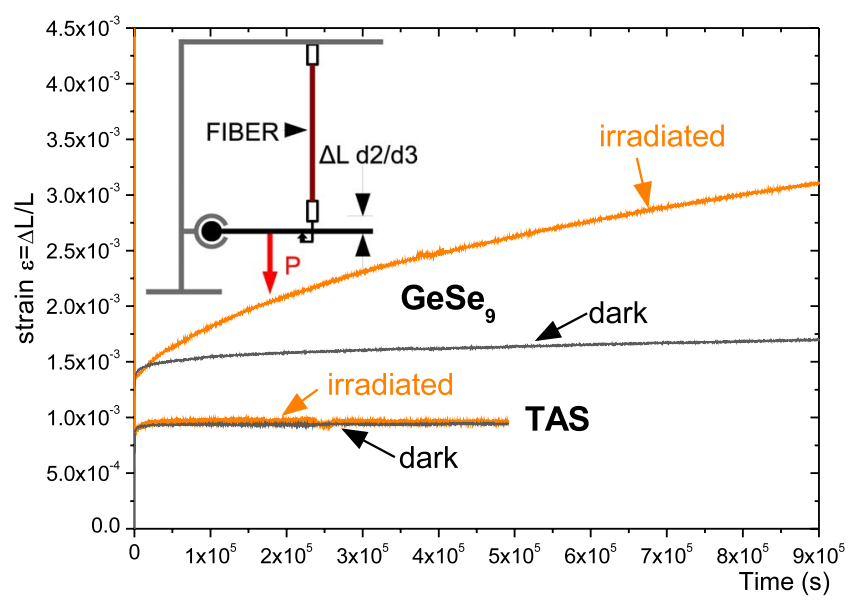

FIG. 2. (Color online) Experimental creep curves for $\mathrm{GeSe}_{9}$ and TAS fibers under dark and irradiated ambiences. On the left, the schematics of the experimental device is drawn. $\mathrm{d} 2$ the distance between the fiber and the carbon tube articulation, $\mathrm{d} 3$ the carbon tube length.

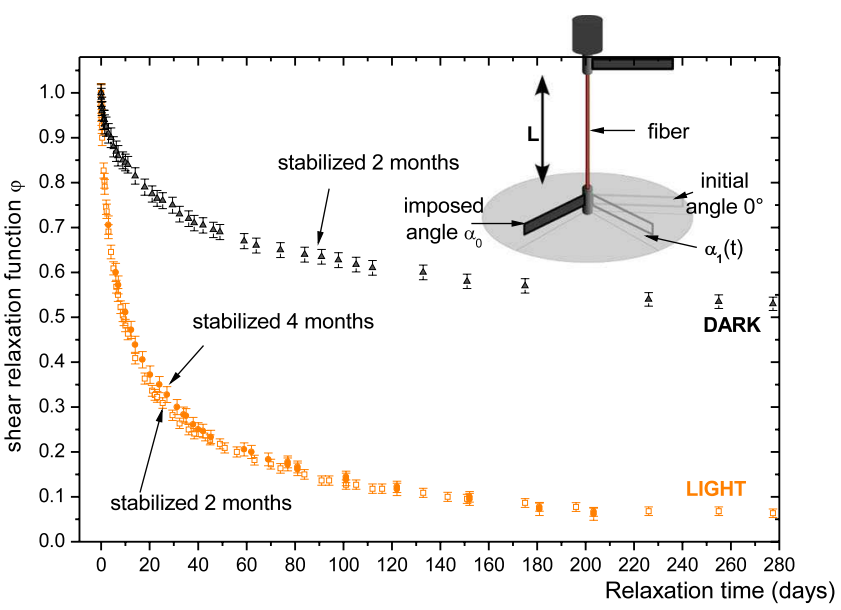

FIG. 3. (Color online) Shear relaxation curves of $\mathrm{GeSe}_{9}$ fibers under dark and irradiated ambiences, and schematics of torsion relaxation test.

\section{Shear relaxation test}

In the shear relaxation test (see Fig. 3), one end of the fiber is fixed in its holder and the other end is submitted to a rotation with an angle $\alpha_{0}$. An imposed constant shear strain $\gamma_{0}=d \alpha_{0} /(2 L)$ derives, where $L$ is the fiber length and $d$ its diameter. Such a test is called "shear relaxation test" since the strain is constant and the stress decreases. When the fiber is released at a relaxation time " $t$," the end does not rotate back to its original position, since an angle $\alpha_{1}$ remains. Consequently, the fiber instantly rotates back of an angle $\alpha_{e}(t)$ $=\alpha_{0}-\alpha_{1}(t)$, which is defined as the elastic recovery due to elastic strain: $\gamma_{e}(t)=d \alpha_{e}(t) /(2 L)$. As the elastic shear strain is proportional to the stress $(\sigma)$ (Hooke's law) and $\sigma$ to the relaxation function, at a time $t$, the shear relaxation function $\varphi$ is simply defined as

$$
\varphi(t)=\frac{\sigma(t)}{\sigma(0)}=\frac{\alpha_{e}(t)}{\alpha_{0}}=1-\frac{\alpha_{1}(t)}{\alpha_{0}} .
$$

\section{B. Results}

In order to investigate the contribution of the glass band gap on the low intensity photofluidity, creep tests were performed on $\mathrm{GeSe}_{9}$ fibers and TAS fibers. Results in Fig. 2 clearly show that light has no effect on TAS fibers. This is because most of the light is absorbed near the surface and produces only surface effects: ${ }^{29}$ the light used has a very low penetration in TAS glass because it corresponds to energies far higher than the Tauc energy of this glass. It is important to note that temperature measurements, with an infrared thermography camera (Thermacam FLIR Systems) with a sensitivity of $2{ }^{\circ} \mathrm{C}$, showed no warming due to absorption susceptible to enhance the fluidity. Therefore, at this low intensity, we can assume that: (i) the observed changes in chalcogenide glasses are only attributed to wavelengths corresponding to subband-gap light and (ii) no thermal effects are present. For $\mathrm{GeSe}_{9}$ (Fig. 2) the irradiation effect is clearly visible: the strain increases faster under irradiation than in the dark. This test clearly demonstrates the existence of 


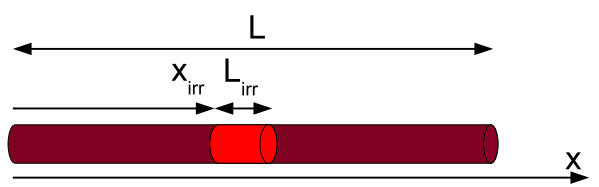

FIG. 4. (Color online) Schematic representation of an irradiated fiber.

photofluidity at low exposure. However, the contribution of fiber elongation due to photoexpansion on the total elongation observed during creep under irradiation is unknown. Moreover, these measurements were performed on a freshly drawn fiber and measuring the viscosity of a fresh glass involves a high uncertainty because the viscosity increases throughout the creep test due to natural enthalpy relaxation. ${ }^{30,31}$ Nevertheless, using the slope of the curve plotted in Fig. 2, the following shear viscosities can be estimated: $5 \times 10^{15} \mathrm{~Pa} \mathrm{~s}$ under irradiation and $4 \times 10^{16} \mathrm{~Pa} \mathrm{~s}$ in the dark.

In order to avoid these issues, $\mathrm{GeSe}_{9}$ fibers were submitted to a dedicated torsion test (see Fig. 3) derived from a previous bending test. ${ }^{23}$ In the torsion configuration, superficial photoexpansion processes should have no effects on the measured viscosity. Finally, in order to circumvent any effect of enthalpy relaxation, fibers dedicated to torsion tests were stored during 2 to 4 months under irradiation in order to allow them to fully stabilize before the measurement. ${ }^{24}$

The relaxation functions obtained are plotted in Fig. 3. First, we note that the fibers are stabilized because their shear relaxation functions are identical after 2 and after 4 months. ${ }^{32}$ As expected for photofluidity, the fibers under irradiation relax faster than those in the dark. The shear viscosity $(\eta)$ is defined as $\eta=\mu \int_{0}^{+\infty} \varphi(t) d t,{ }^{33}$ with $\mu$ the shear elastic modulus in the dark (see Table I). Measurements of shear elastic modulus do not indicate any change under such a low light exposure. The shear viscosity values calculated are: $10^{16.5} \mathrm{~Pa}$ s under irradiation and $10^{18.6} \mathrm{~Pa} \mathrm{~s}$ in the dark by extrapolating the available data. Note that the viscosity in the dark is not the viscosity under thermodynamic equilibrium but the viscosity after a dynamic equilibrium obtained by irradiation. These two equilibriums are different. ${ }^{34}$ The viscosity obtained is a viscosity of a state called "illuminated state" by Tanaka 35 as opposed to the "transitory" one (under irradiation). The results of Figs. 2 and 3 clearly indicate that photofluidity can be induced even at very low intensity. This observation calls for a reassessment of the mechanism and the origin of photofluidity.

\section{HIGH-INTENSITY IRRADIATION}

\section{A. Experiments}

For high-intensity tests, a fiber of length $L$ is irradiated from an abscissa $x_{i r r}$ along a length $L_{i r r}$ as schematized in Fig. 4. An irradiated fiber can be considered as a viscoelastic composite with two components: the irradiated volume and the unirradiated one. The mechanical behavior of this composite can be described by an analytic solution. We have first to determine the solution for an elastic composite. In a fiber submitted to a torque $M_{T}$, the stress can be written as

$$
\sigma(r)=\frac{M_{T}}{I_{P}} r
$$

$r$ is the distance between a considered point in the fiber volume and the neutral axis of the fiber and $I_{P}$ the polar quadratic moment of the fiber. This expression illustrates that the stress is constant all along the fiber length. The shear modulus $\mu$ of the fiber is constant in each section at a length $x$ and defined as: $\mu(x)=\mu_{\text {dark }}$ for $0<x<x_{i r r}$ and $x_{i r r}+L_{i r r}<x<L$, corresponding to the unirradiated volume; and $\mu(x)=\mu_{i r r}$ for $x_{i r r}<x<x_{i r r}+L_{i r r}$, corresponding to the irradiated volume. Considering the section at $x=0$ as fixed, the rotation $\alpha(x)$ of a fiber section at a abscissa $x$ is defined as

$$
\sigma(r)=r \mu(x) \frac{d \alpha(x)}{d x}
$$

So that we obtain, for $\alpha(L)=\alpha_{0}$,

$$
\sigma(r)=r \alpha_{0} \frac{\mu_{d a r k} \mu_{i r r}}{\mu_{i r r}\left(L-L_{i r r}\right)+\mu_{d a r k} L_{i r r}} .
$$

The stress does not depend on the length $x_{i r r}$. The solution for a viscoelastic composite submitted to a constant rotation $\alpha_{0}$ is given using the well-known Lee-Mandel correspondence principle, ${ }^{36}$ by applying the method of functional equations. Let substitute the shear elastic moduli by $G_{d a r k}$, the time-dependent shear relaxation modulus of the unirradiated volume and $G_{i r r}$, the time-dependent shear relaxation modulus of the irradiated volume and let denote $f^{*}$ the Laplace-Carson transform of a function $f$ and $p$ the Laplace variable. By substituting each time-dependent function, including the stress, by its Laplace-Carson transform, we have then

$$
\sigma^{*}(p, r)=r \alpha_{0} \frac{G_{d a r k}^{*}(p) G_{i r r}^{*}(p)}{G_{i r r}^{*}(p)\left(L-L_{i r r}\right)+G_{d a r k}^{*}(p) L_{i r r}} .
$$

We can normalize the stress by the Laplace-Carson transform of its expression at $t=0$, corresponding to the stress for an elastic composite given by Eq. (4). This normalization gives an apparent shear relaxation function $\varphi_{a p p}$,

$$
\begin{aligned}
\varphi_{\text {app }}^{*}(p) & =\frac{\sigma^{*}(p, r)}{\sigma^{*}(r)} \\
& =\frac{G_{\text {dark }}^{*}(p) G_{i r r}^{*}(p)\left[L_{i r r}\left(\mu_{\text {dark }}+\mu_{i r r}\right)+L \mu_{i r r}\right]}{\left[G_{i r r}^{*}(p)\left(L-L_{i r r}\right)+G_{\text {dark }}^{*}(p) L_{i r r}\right] \mu_{\text {dark }} \mu_{i r r}} .
\end{aligned}
$$

$\mathrm{GeSe}_{9}$ fibers, $300 \mu \mathrm{m}$ in diameter, and $120 \mathrm{~mm}$ in length were first submitted to a shear relaxation test without laser irradiation, following the procedure described in Sec. III A 2, in order to identify the shear relaxation function $\varphi$ in the dark, Recalling that the shear relaxation modulus is: $G(t)$ $=\mu \varphi(t)$. The shear relaxation modulus in the dark $G_{\text {dark }}$ can be fitted by 
TABLE II. Parameters of generalized Maxwell's model [Eq. (7)] describing the relaxation function of an unirradiated $\mathrm{GeSe}_{9}$ fiber.

\begin{tabular}{ccc}
\hline \hline$w$ & $\begin{array}{c}\tau_{\text {dark }}^{1} \\
(\text { min })\end{array}$ & $\begin{array}{c}\tau_{\text {dark }}^{2} \\
(\text { min })\end{array}$ \\
\hline 0.10 & 1.01 & 10000 \\
\hline \hline
\end{tabular}

$$
G_{\text {dark }}(t)=\mu_{\text {dark }}\left[w \exp \left(-\frac{t}{\tau_{\text {dark }}^{1}}\right)+(1-w) \exp \left(-\frac{t}{\tau_{\text {dark }}^{2}}\right)\right]
$$

We can then identify $w, \tau_{\text {dark }}^{1}$, and $\tau_{\text {dark }}^{2}$ in Eq. (7). Fitted values are given in Table II.

We do the assumption that the shear relaxation modulus of the irradiated volume can be written as

$$
G_{i r r}(t)=\mu_{i r r} \exp \left(-\frac{t}{\tau_{i r r}}\right) \text {. }
$$

Using the expressions of $G_{d a r k}$ and $G_{i r r}$, the MATHEMATICA software can provide an analytic solution for the inverse Laplace-Carson of the expression of $\varphi_{a p p}^{*}(p)$ of Eq. (6). The theoretical normalized stress $\varphi_{a p p}$ is a simple function of $\tau_{i r r}$ in the sense that, for $t>0, \varphi_{a p p}$ monotonically increases with $\tau_{i r r}$. This expression is too long to be developed here. All these calculations have been controlled by modeling the problem using finite-element analyses (CAST3M-CEA, www-cast3m.cea.fr) with generalized Maxwell models: analytic and numerical solutions are in perfect accordance.

The fiber is then irradiated using a tunable cw Ti:sapphire laser with a known spot profile shown in Fig. 5. The laser is polarized perpendicularly to the fiber. As the strain tensor is purely deviatoric, there is no volume change, at least mechanically induced, of the irradiated volume. Moreover, deviatoric strain does not induce any elongation so that the volume under irradiation is always the same all along the

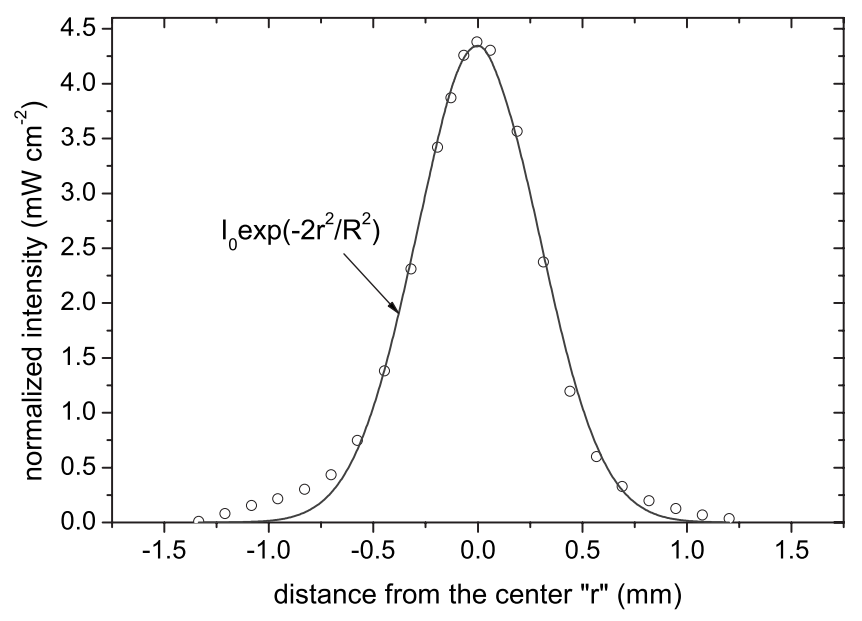

FIG. 5. Normalize profile of the laser spot. The intensity $I$ is normalized so that the power defined as $\int_{0}^{+\infty} \int_{0}^{2 \pi} I(r) r d \theta d r$ is equal to $1 \mathrm{~mW} . R$ is the spot radius usually defined as the distance $r$ for which the intensity is $I_{0} / e^{2}$ with $I_{0}$ the maximum intensity at $r=0$. test. Structural orientation in the Se-rich fibers was shown to be minimal either by static NMR or polarized Raman hence its effect should not be very significant. But, even if a transversal isotropy exists, the shear relaxation test, as the shear stress is applied along planes perpendicular to the fiber symmetry axis, does not aggravate this anisotropy, as tensile test does. For the same reason, the shear relaxation test does not restrain the relaxation of this anisotropy that can be produced through photoinduced processes.

The radius of the spot is $R=0.58 \mathrm{~mm}$. Fibers are first irradiated at a $790 \mathrm{~nm}$ wavelength, to investigate the power sensitivity of photofluidity. This wavelength corresponds to $80 \%$ of the maximum transmission and to a penetration higher than $1 \mathrm{~mm}$ and induces bulk effects in fiber. The powers used were 50, 70, 90, and $110 \mathrm{~mW}$ (intensities absorbed ranging between $1.5 \mathrm{~W} \mathrm{~cm}^{2}$ and $5.6 \mathrm{~W} \mathrm{~cm}^{2}$, respectively). In order to investigate the wavelength sensitivity of photofluidity, fibers are then irradiated at a constant power (110 $\mathrm{mW}$ ) using various wavelengths: 785, 790, 795, and $800 \mathrm{~nm}$.

The fiber is irradiated at a constant position $x_{i r r}$ and submitted to a torsion test with a constant rotation $\alpha(L)=200^{\circ}$ at its end. As shown in Eq. (1), the experimental normalized stress $\left(\varphi_{\text {app }}\right)$ is a function of $\alpha_{e}$. Then, measuring $\alpha_{e}, \tau_{i r r}$ can be identified thanks to Eq. (6), using a Newton-Raphson scheme by minimizing the difference between the experimental normalized stress and the theoretical normalized stress. As $\varphi_{a p p}$ is a simple function of $\tau_{i r r}$, the minimization gives an unique solution. It is uneasy to measure the elastic properties under subband gap irradiation so that $\mu_{i r r}$ is unknown. As pointed out by Tanaka et al., ${ }^{35}$ measurements are not reproducible. Here, we suppose, as the viscosities observed under irradiation are close to those observed at $T_{g}$, that the shear modulus under irradiation will be the same as the one measured at $T_{g}$ in the Ge-Se system. As $\mu_{\text {dark }}$ is the shear modulus at room temperature, we consider that $0.85 \mu_{\text {dark }}<\mu_{\text {irr }}<\mu_{\text {dark }}$, knowing that the maximum decrease in shear modulus at $T_{g}$ is almost $15 \%$ in Ge-Se system. ${ }^{28}$ The error bar of viscosities takes into account all the range of acceptable values of $\mu_{i r r}$. The length $L_{i r r}$ is considered to be two times the laser spot radius $(R=0.58 \mathrm{~mm})$ and to be affected by an uncertainty equal to the deflection of the arc of a circle of radius $R$ with a chord of $300 \mu \mathrm{m}$ (the fiber diameter). Then, $L_{i r r}=1.07 \pm 0.09 \mathrm{~mm}$.

\section{B. Results}

Figure 6 shows a set of shear relaxation results at constant wavelength as a function of the power for $\mathrm{GeSe}_{9}$ fibers. The power used are 50, 70, 90, and $110 \mathrm{~mW}$ (corresponding to $4.7 \mathrm{~W} / \mathrm{cm}^{2}, 6.6 \mathrm{~W} / \mathrm{cm}^{2}, 8.5 \mathrm{~W} / \mathrm{cm}^{2}$, and $10.4 \mathrm{~W} / \mathrm{cm}^{2}$, respectively). Equation (7) is sufficient to describe the relaxation function in the dark, and Eq. (6) is sufficient to describe the mechanical behavior under irradiation. We must first underline that, considering the wavelength and the power used, the glass structure equilibrates under irradiation after less than $10 \mathrm{~min},{ }^{37}$ and that the experimental duration (up to $5 \mathrm{~h}$ ) is sufficiently long to be representative of the equilibrium state. Experimental results on Ge-Se systems ${ }^{38}$ also suggest that photodarkening saturates after less than $10 \mathrm{~min}$ at the 


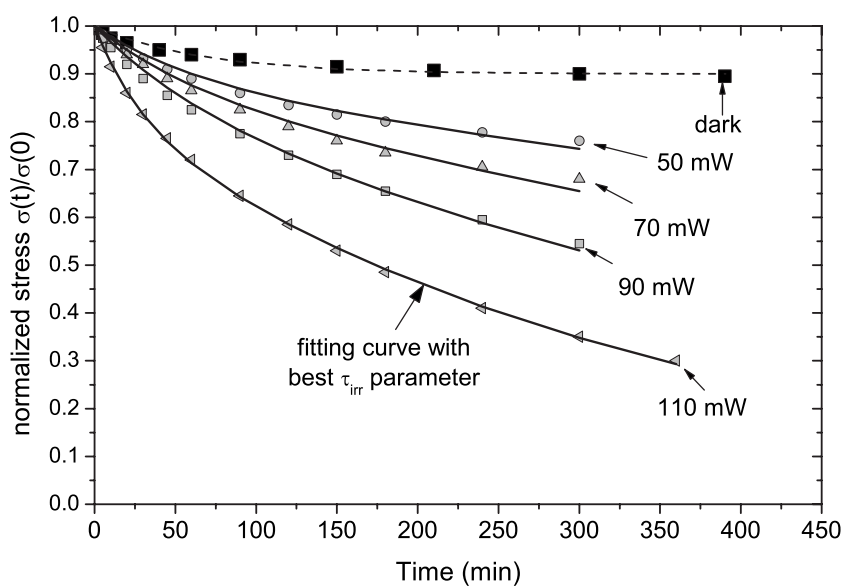

FIG. 6. Normalized stress relaxation during shear tests under irradiation. The dash line fits the relaxation curve in the dark using Eq. (7); the full lines fit the curves under irradiation using Eq. (6).

intensity used here. Figure 7 shows a fiber after a shear relaxation test. From this figure, we clearly see the localization of the shear deformation under the laser spot, and it clearly illustrates how fluid the glass becomes. The shear viscosity is
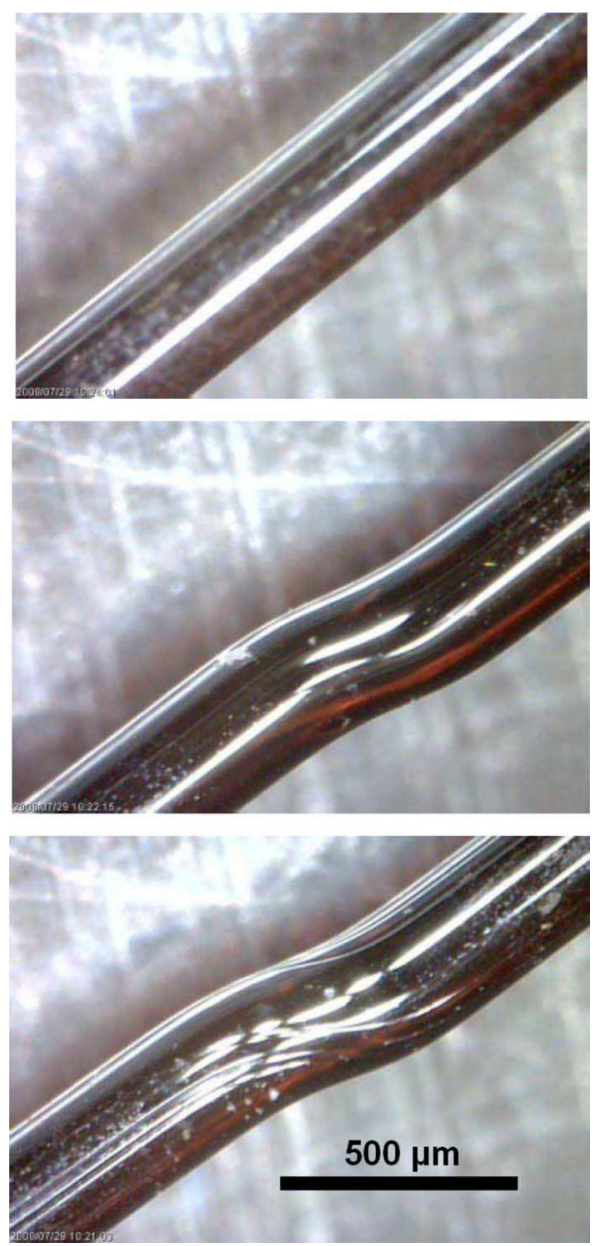

FIG. 7. (Color online) Localization of the shear deformation under the laser spot in a $\mathrm{GeSe}_{9}$ fiber submitted to shear relaxation test under laser irradiation (wavelength: $790 \mathrm{~nm}$ ).

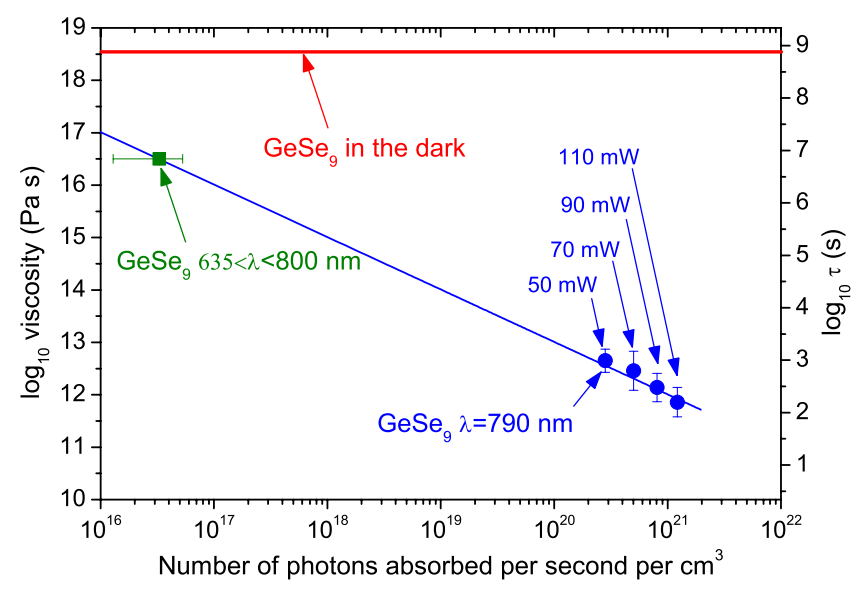

FIG. 8. (Color online) Evolution of the viscosity measured by torsion tests as a function of number of photons absorbed per volume unit in $\mathrm{GeSe}_{9}$ and evolution of the time required to produce a motion of all the atoms in the irradiated volume $(\tau)$. The full line corresponds to the model.

calculated using the relationship $\eta_{i r r}=\mu_{i r r} \tau_{i r r}$ (Maxwell's relationship), taking into account the uncertainty concerning $\mu_{\text {irr }}$ (see Sec. IV A). The viscosities are plotted in Fig. 8. We clearly see here the photoinduced fluidity as depicted by Tanaka et al: $:^{9,12}$ the viscosity decreases as the power increases. The thermally induced viscosity of $\mathrm{GeSe}_{9}$ at the thermodynamic equilibrium is larger than $10^{19} \mathrm{~Pa} \mathrm{~s}$ at room temperature and reaches $10^{12} \mathrm{~Pa} \mathrm{~s}$ at $85^{\circ} \mathrm{C} .{ }^{28}$ Temperature measurements using infrared thermography do not indicate any warming due to irradiation so that the viscosity decrease observed can be considered as purely athermal. ${ }^{39}$ Moreover, investigations made in Ge-Se system ${ }^{40}$ using Raman temperature measurements did not show any temperature increase larger than $3{ }^{\circ} \mathrm{C}$, recalling that an increase of $65^{\circ} \mathrm{C}$ is needed to observe such low viscosities.

In the optical-absorption edge, the absorption decreases as the wavelength increases and moreover, the closer the wavelength is to $\lambda_{g}$, the greater the photodarkening. The viscosities measured under $100 \mathrm{~mm}$ at 785, 790, 795, and $800 \mathrm{~nm}$ are plotted in Fig. 9. As expected, as the wavelength increases, since the absorption decreases, the viscosity increases because fewer photons are absorbed.

\section{A model for photoinduced fluidity}

It is well accepted that the photofluidity effect is the result of successive and dynamic photoexcitations of valence electrons leading to bond reformations in the glass network..$^{5,41,42}$ The dynamic nature of this process implies that the power dependence is a key factor in understanding its mechanism. Here we propose a quantitative model for the photoinduced fluidity which is consistent with viscosity values measured over a wide range of irradiation powers.

We first consider the quantity of photons absorbed by the fiber per second. Integrating the spot intensity profile fitted by $I(r)=I_{0} \exp \left(-\frac{2 r^{2}}{R^{2}}\right)$, as illustrated in Fig. 5 , between adequate boundaries, we can estimate the power $P_{r}$ received by the fiber as a function of the power of the laser. The irradi- 


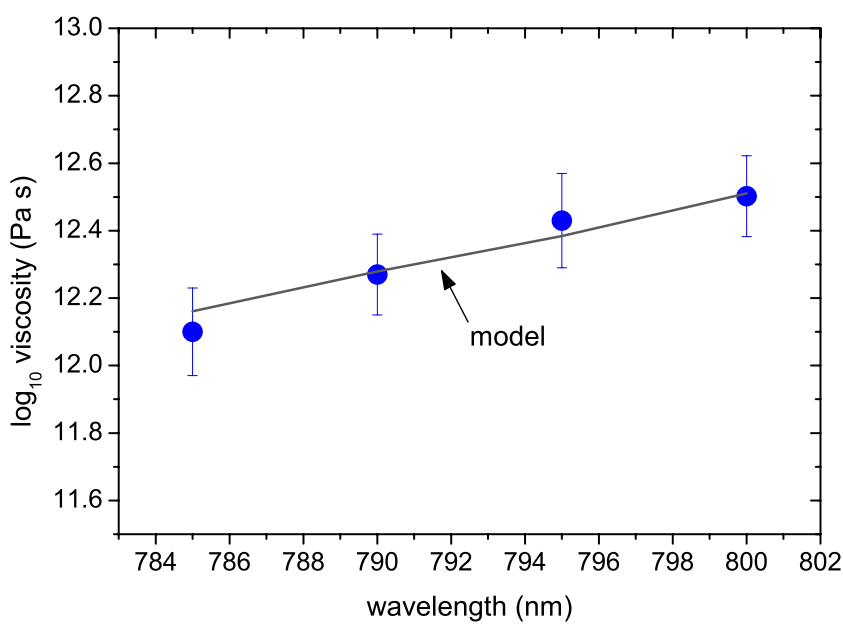

FIG. 9. (Color online) Evolution of the viscosity measured by torsion test as a function of the irradiation wavelength in $\mathrm{GeSe}_{9}$ under $100 \mathrm{~mW}$. The full line corresponds to the model.

ated area on the fiber is delimited by a width $d$, the fiber diameter and a length $2 R$, the spot diameter. $\phi$ being the ratio $\frac{P_{r}}{P}$,

$$
\phi=\frac{\int_{-d / 2}^{d / 2} \int_{-R}^{R} I_{0} \exp \left[-\frac{2\left(x^{2}+y^{2}\right)}{R^{2}}\right] d x d y}{\int_{0}^{+\infty} \int_{0}^{2 \pi} I_{0} \exp \left(-\frac{2 r^{2}}{R^{2}}\right) r d \theta d r}
$$

We obtain $\phi=0.377$ so that $P_{r}=0.377 P$. The reflectance of the $\mathrm{GeSe}_{9}$ glass is almost $0.225,{ }^{26}$ so that we can consider that a ratio of $\rho=1-0.225=0.775$ of the power is transmitted. Knowing the power $P_{r}$ transmitted and the wavelength $\lambda$, we can calculate the number of photons transmitted per second, recalling $E=\frac{h C}{\lambda}$ is the photon energy: $\rho \frac{\phi P}{E}$ ( $h$ the Planck's constant and $C$ the speed of light). The irradiation induces photodarkening which is a function of the power of the laser, increasing the absorption. The absorption $(a)$ in photodarkening saturation condition has been measured at $50,70,90$, and $100 \mathrm{~mm}$ on a 300- $\mu \mathrm{m}$-thick sample at 790 $\mathrm{nm}$. They are listed in Table III. The absorption can be fitted as a function of the power $P$ (in watt) with a better than $2 \%$ accuracy as: $a=6.09 \exp (14.34 P)$. We have also measured

TABLE III. Absorption at photodarkening saturation at $790 \mathrm{~nm}$ in a $300-\mu \mathrm{m}$-thick $\mathrm{GeSe}_{9}$ glass sample. The absorption at "0" $\mathrm{mW}$ is the absorption at short time, when the photodarkening has not been induced.

\begin{tabular}{cc}
\hline \hline $\begin{array}{c}\text { Power } \\
(\mathrm{mW})\end{array}$ & $\begin{array}{c}\text { Absorption } \\
\left(\mathrm{cm}^{-1}\right)\end{array}$ \\
$\sim 0$ & 6.2 \\
50 & 12.3 \\
70 & 16.2 \\
90 & 22.6 \\
110 & 25.8 \\
\hline
\end{tabular}

TABLE IV. Absorption at photodarkening saturation at $100 \mathrm{~mW}$ in a $300-\mu \mathrm{m}$-thick $\mathrm{GeSe}_{9}$ glass sample.

\begin{tabular}{cc}
\hline \hline $\begin{array}{c}\text { Wavelength } \\
(\mathrm{nm})\end{array}$ & $\begin{array}{c}\text { Absorption } \\
\left(\mathrm{cm}^{-1}\right)\end{array}$ \\
\hline 785 & 41.5 \\
790 & 25.8 \\
795 & 17.9 \\
800 & 12.6 \\
\hline \hline
\end{tabular}

the absorption at photodarkening saturation on $300-\mu$ m-thick samples at $100 \mathrm{~mW}$ using the following wavelengths 785 , 790, 795, and $800 \mathrm{~nm}$. The corresponding absorption are given in Table IV.

Using the absorption, we can estimate the number of photons absorbed per second through the length $D$ they cross. $D$ is chosen to be $236 \mu \mathrm{m}$, the mean thickness of the fibers (i.e., two times the algebraic mean of an half circle of diameter $300 \mu \mathrm{m})$. The ratio of photons absorbed is given by the absorption and the length $D: \kappa=1-\exp (-a D)$. The number of photons absorbed per second by the fiber is $\frac{\rho \phi P}{E} \kappa$, recalling $a$ is given in Tables III and IV.

Now, we consider that photoinduced fluidity is due to photon absorptions, creation of self-trapped excitons and nonradiative recombinations of excited electron-hole pairs. The creations of self-trapped excitons and their recombinations induce diffusive atomic motions. ${ }^{5,41,42}$ Fritzsche has suggested that these excitons could be VAPs, ${ }^{41}$ and it already exists a model that describe VAPs as structures that can easily diffuse. ${ }^{43}$ Most of models for viscous flow, and especially the well-known Adam-Gibbs equation, ${ }^{44}$ assume that the stress relaxation time $(\tau)$ is inversely proportional the fraction of subsystems that are in a state permitting the rearrangements involved in viscous flow. Lets consider these subsystems as being the excitons or VAPs in the present case, each VAP being created by a single photon. Then, the number of subsystems created per second is proportional to the number of photons absorbed per second $\left(\frac{\rho \phi P}{E} \kappa\right)$. The $n \frac{\rho \phi P}{E} \kappa / N$ ratio is the fraction of subsystems that are in state permitting rearrangement, $N$ being the number of atoms in the irradiated volume and $n$ a proportionality factor. This proportionality factor $n$ involves the energy barrier for diffusive motion of VAPs, the number of atoms per subsystems (VAP) and the probability that excitons diffuse instead of going back to their ground state. If we assume that these parameters are not sensitive to the laser power or wavelength, they remain unchanged whatever the irradiation conditions. We will simply consider that the stress relaxation time $\tau$ corresponding to $\tau_{i r r}$ in Eq. (8) is, as in the AdamGibbs equation, inversely proportional to the fraction of state permitting rearrangements: $n \frac{\rho \phi P}{E} \kappa / N$. We will not take into account the thermally induced relaxation because its characteristic time is over 70 years $\left(10^{19} \mathrm{~Pa} \mathrm{~s} / 4.6 \mathrm{GPa}\right)$ so that the thermally induced relaxation is negligible at room temperature. It corresponds to the assumption that no other subsystems than VAPs are in states permitting equivalent rearrangements. The volume of the fiber delimited by $L_{i r r}$ $=1.07 \mathrm{~mm}$ contains $N=2.57 \pm 0.01 \times 10^{18}$ atoms, consider- 
TABLE V. Parameters of Eq. (10)

\begin{tabular}{lc}
\hline \hline Parameter & Symbol \\
\hline Power of the laser source (W) & $P$ \\
Ratio of unreflected light & $\rho$ \\
Ratio of incident light on the fiber & $\phi$ \\
Ratio of photons absorbed & $\kappa$ \\
Photon energy & $E$ \\
Number of atoms in the irradiated volume & $N$ \\
Photon "efficiency" & $n$ \\
\hline \hline
\end{tabular}

ing the number of atoms per millimeter length (see Table I). The stress relaxation time $\tau$ evaluated through the number of photons absorbed is

$$
\begin{aligned}
\tau & =\frac{N}{n}\left(\frac{\rho \varphi P \kappa}{E}\right)^{-1} \\
& =\frac{2.57 \times 10^{18}}{n}\left\{0.775 \frac{0.377 P \lambda}{h C}[1-\exp (-0.0236 a)]\right\}^{-1} .
\end{aligned}
$$

All the parameters of this equation are summarized in Table $\mathrm{V}$ for sake of clarity. Then, we can estimate the shear viscosity from $\tau$ as $\eta=\tau \mu_{i r r}$, and compare it with the experimental viscosities obtained at a constant wavelength (790 $\mathrm{nm})$. The best fit is obtained for $n=0.2 \pm 0.05$. The model is plotted along with experimental data in Fig. 8. In Sec. III B we have reported the viscosity of a $\mathrm{GeSe}_{9}$ fiber under very low intensity using a large spectra of wavelengths. Using the transmission spectra shown in Fig. 1, we can calculate the absorption for all the wavelengths from $\lambda_{g}$ to $800 \mathrm{~nm}$. When $\lambda<\lambda_{g}$, the absorption can be considered as complete since $\exp (-a D)$ is $\sim 0$ when $a>1000 \mathrm{~cm}^{-1}$. Above $800 \mathrm{~nm}$, the fluorescent light does not emit significant light intensity and the absorption becomes too low. Knowing the intensity received by the fiber at each wavelength, we can calculate the number of photons absorbed per second by the fiber. The photodarkening at such a low intensity is considered to be negligible. The fiber absorbs $2.5 \pm 1 \times 10^{13}$ photons per second per centimeter length and the fiber contains $2.40 \times 10^{19}$ atoms per centimeter length (see Table I). So that, considering the same $n$ factor, and that the shear modulus is not consequently affected by light and is $4.6 \mathrm{GPa}$, the viscosity estimated thanks to the model range from $10^{16.8} \mathrm{~Pa} \mathrm{~s}$, if we only take into account the wavelengths above $\lambda_{g}$, to $10^{16.3} \mathrm{~Pa} \mathrm{~s}$, if we take into account all the wavelengths. The horizontal error bar in Fig. 8 corresponds to these two hypotheses. We calculate the viscosity using all the wavelengths only to illustrate that the correspondence observed here is not fortuitous and is not due to the wavelengths taken into account. Note that the viscosity predicted by the model is two times higher than the experimental one $\left(10^{16.5} \mathrm{~Pa} \mathrm{~s}\right)$, but 60 times lower than the viscosity in the dark. So that we can consider that a good agreement exists between the model and the experimental data. It shows that the model is able to take into account the power dependence over 5 orders of magnitude (from $165 \mu \mathrm{W} / \mathrm{cm}^{2}$ to $10 \mathrm{~W} / \mathrm{cm}^{2}$ ).

The viscosities under laser irradiation at 785, 790, 795, and $800 \mathrm{~nm}$ have been also calculated with $P=100 \mathrm{~mW}$. The viscosities predicted by the model are plotted in Fig. 9 with experimental viscosities. We clearly see that the model gives a very good prediction of the sensitivity to wavelength. Hence, with a single fitting parameter $n$, the model is able to predict both the sensitivity to the power and to the wavelength with a good accuracy without any adjustment.

The fitting parameter $n$ is a proportionality factor between viscosity and the fraction of photoinduced states permitting rearrangements. It is expected that $n$ should be small since each given photoexcitation event has a moderate probability of inducing a structural rearrangement. Indeed, accepted models for photostructural changes ${ }^{3,5,45}$ are based on the principle that a photoexcited electron can either radiate back into its original state, or produce a new state such as a selftrapped exciton. This state can then either relax back to its original configuration or produce a net photostructural rearrangement. If we consider that this state corresponds to a VAP, the probability of producing a net rearrangement is low. ${ }^{43}$ Hence, the probability of a single photon absorption to induce an atomic rearrangement is expected to be significantly low. Therefore, $n$ can be regarded as the photon efficiency for inducing one atomic rearrangement. Explicitly, Eq. (10) suggests that the time constant associated with photoinduced viscous flow is equivalent to the time required to induce motion of every photoinduced structural defect in the irradiated volume. In other words, the photoinduced viscous flow is a summative process of local constraint release under an applied stress. It is then expected that this mechanism should be effective even at very low intensities. Indeed, Fig. 8 shows that Eq. (10) appears to hold over more than 5 orders of magnitudes in light intensity.

\section{MEAN COORDINATION EFFECT}

Previous studies ${ }^{46}$ have described the effect of mean coordination $(\langle r\rangle)$ of the glass network on photoinduced behaviors. The mean coordination number of $\mathrm{Ge}_{x} \mathrm{Se}_{1-x}$ are given in Table I. Photoexpansion, photodarkening, and photoinduced structural relaxation are shown to be dependent on the mean coordination. ${ }^{24}$ All these photoinduced effects vanish as the mean coordination increases if the glasses are irradiated in the same conditions, i.e., with the same power and with wavelengths corresponding to the same absorption.

In order to investigate the effect of mean coordination on photofluidity, $\mathrm{GeSe}_{4}$ and $\mathrm{GeSe}_{3}$ fibers, $300 \mu \mathrm{m}$ in diameter, were irradiated with $777 \mathrm{~nm}$ and $763.5 \mathrm{~nm}$ wavelengths, respectively. At these wavelengths, their absorption is equivalent to that of $\mathrm{GeSe}_{9}$ at $790 \mathrm{~nm} .{ }^{24}$ We followed exactly the same experimental protocol than for $\mathrm{GeSe}_{9}$. Fibers have been submitted to torsion tests and their viscosities have been measured using the protocol defined in Sec. IV A with different powers: 140, 150, 160, and $170 \mathrm{~mW}$ for $\mathrm{GeSe}_{4}$, and $150,170,180$, and $190 \mathrm{~mW}$ for $\mathrm{GeSe}_{3}$. The spot profile is unchanged. The shear moduli of $\mathrm{GeSe}_{4}$ and $\mathrm{GeSe}_{3}$ glasses are given in Table I. The experimental viscosities are plotted in Fig. 10. Note that the viscosity of $\mathrm{GeSe}_{4}$ at room tempera- 


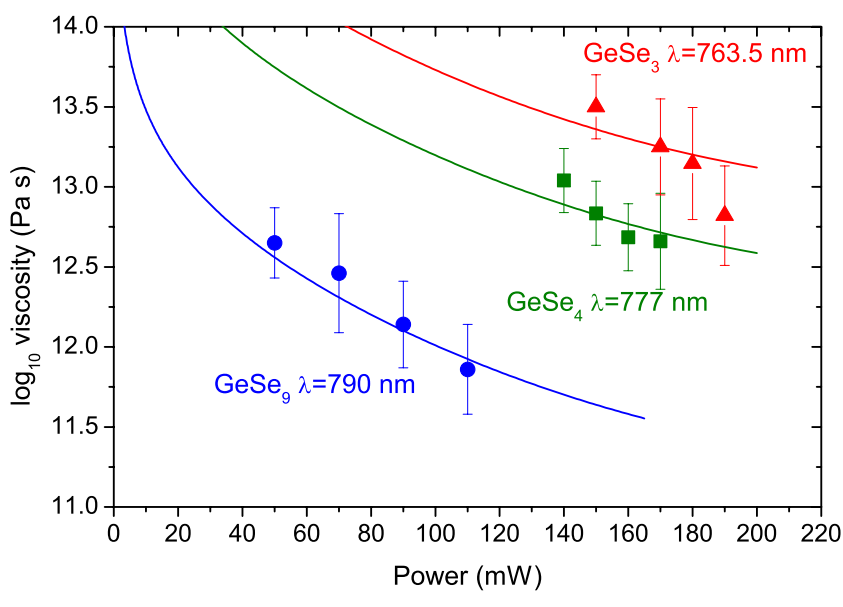

FIG. 10. (Color online) Evolution of the viscosities measured by torsion tests as a function of the power of the light source in $\mathrm{Ge}-\mathrm{Se}$ system. The full lines correspond to the model.

ture is supposed to be over $10^{25.5} \mathrm{~Pa} \mathrm{~s}$, corresponding to a relaxation time larger than hundred millions of years, that of $\mathrm{GeSe}_{3}$ is supposed to be even higher. $\mathrm{GeSe}_{4}$ glass reaches viscosities below $10^{13} \mathrm{~Pa}$ s only above $150{ }^{\circ} \mathrm{C}$ for thermally activated processes. ${ }^{28}$ From this observation, we can reasonably conclude that no thermal effect has to be taken into account.

The model defined by Eq. (10) can be applied to $\mathrm{GeSe}_{4}$ and $\mathrm{GeSe}_{3}$ fibers, taking into account the absorption due to photodarkening and its sensitivity to the power of the laser. $\mathrm{GeSe}_{4}$ and $\mathrm{GeSe}_{3}$ contain $2.61 \times 10^{18}$ atoms in the irradiated volume with $L_{i r r}=1.07 \mathrm{~mm}$ (see Table I). The best fit is obtained for $n=0.013 \pm 0.005$ for $\mathrm{GeSe}_{4}$ and $n=0.005 \pm 0.001$ for $\mathrm{GeSe}_{3}$. These low $n$ values compared to those obtained for $\mathrm{GeSe}_{9}$ illustrate that the photosensitivity decreases as the mean coordination increases. Concerning the $\mathrm{GeSe}_{3}$ fibers, it seems that a point is not in accordance with the model: it can be explained by a larger uncertainty due to the high viscosity measured.

The number of constraints per atoms $N_{c}$ (Ref. 47) increases as the mean coordination increases: $N_{c}=5 / 2\langle r\rangle-3$. The ideal number of constraints is equal to 3 , the number of degrees of freedom, which corresponds to $\langle r\rangle=2.4$. For higher mean coordination values, the glass is overconstrained, in the sense that the number of constraints is higher than the number of degrees of freedom. It is then expected that, if a photon absorption induces bond breaking or bond changes, ${ }^{6,41}$ it is likely to have a low impact on overconstrained structure as even with a decrease in local coordination, the number of constraints per atoms will still be higher than the degrees of freedom and structural reorganization will therefore be limited by the network rigidity. A large fraction of photons absorbed will then be inefficient in producing atomic motions so that we can expect $n$ to be lower as it is observed experimentally. Conversely, in underconstrained networks, the number of constraints per atoms is lower than the number of degrees of freedom, so that we can expect that a bond breaking event has a greater probability of inducing an atomic rearrangement and $n$ is therefore higher.

El Gharras et al. ${ }^{48}$ have done a similar interpretation of the composition dependence of the photoconductivity in

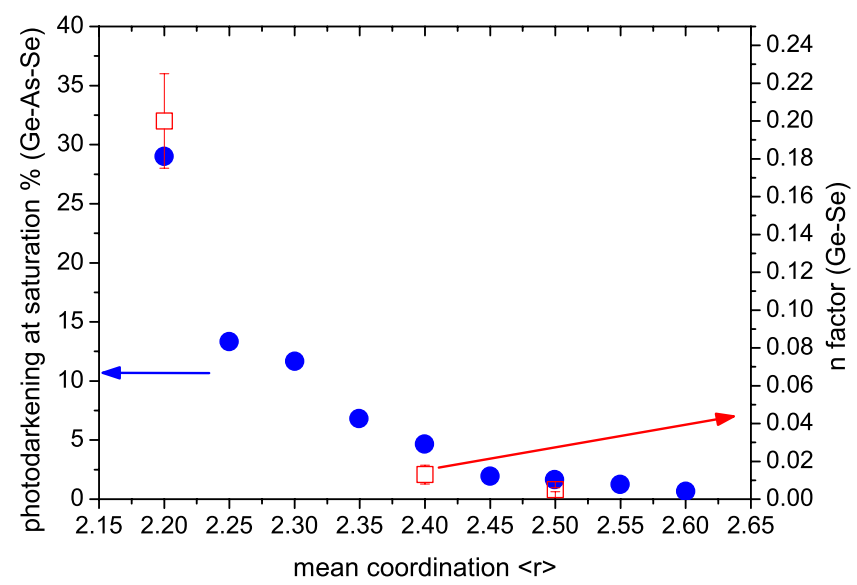

FIG. 11. (Color online) Evolution of the photodarkening at saturation under subband-gap light as a function of $\langle r\rangle$, with wavelengths corresponding to equivalent absorption $\left(1.6 \mathrm{~cm}^{-1}\right.$ without photodarkening) in all $\mathrm{Ge}_{x} \mathrm{As}_{x} \mathrm{Se}_{1-2 x}$ compositions (Ref. 46) and evolution of the photon efficiency $n$ with $\langle r\rangle$ in Ge-Se.

Ge-Se films. They have shown that increasing the Ge content decreases the photoconductivity whatever the photon energy. They have attributed the photoinduced effects to the soft and Se rich regions in Ge-Se glasses. The decrease in photoinduced effects, as the Ge content increases, can also be attributed to the large decrease in the density of defect centers produced by the addition of $\mathrm{Ge} .^{49}$

\section{DISCUSSION}

Most models of photostructural changes ${ }^{5,45}$ suggest that a dynamic equilibrium exists between an increase in the local energy of configuration due to photoinduced events and a decrease in this energy due to thermal relaxation. More recent studies $^{34}$ show that the dynamic equilibrium results from the competition between photoexcitation and optically induced relaxation. As photoinduced events allow atomic mobilities, they enable structural relaxation. In the present model for photofluidity this equilibrium is reflected and quantified by the photon efficiency parameter $n$. The $n$ factor reflects the probability that a photoexcitation event will result in a net structural change. This factor is the only fitting parameter necessary in Eq. (10) to accurately describe the power and wavelength dependence of the photoinduced viscosity when considered as the cumulative motion of all atoms in the irradiated volume. It has previously been shown that the efficiency of photostructural changes greatly depend on the mean coordination of the glass network. ${ }^{46}$ The extent of photodarkening in the Ge-As-Se system has been plotted in Fig. 11 as a function of $\langle r\rangle$ along with the values of $n$ obtained in this study for $\langle r\rangle=2.2,2.4$, and 2.5 in the Ge-Se system. This figure shows a good correlation between the extent of photodarkening at saturation and the factor $n$. This correlation is expected since the photodarkening can be regarded as a measure of the concentration of photoinduced defects in the structure and should therefore be proportional to the probability of creating these defects and hence to $n$ (all other irradiation conditions being equal). This illustrates that 
TABLE VI. $\mathrm{As}_{2} \mathrm{~S}_{3}$ parameters for the model. * length along the light path.

\begin{tabular}{lc}
\hline \hline Properties & $\mathrm{As}_{2} \mathrm{~S}_{3}$ \\
\hline Spot radius $^{\mathrm{a}}$ & $56 \mu \mathrm{m}$ as $10 \mathrm{~mW} \rightarrow 100 \mathrm{~W} / \mathrm{cm}^{2}$ \\
Sample size $^{\mathrm{a}}$ & $5 \times 0.2^{*} \times 0.05 \mathrm{~mm}^{3}$ \\
Irradiated volume $^{-6} \mathrm{~cm}^{3}$ \\
Density & $3.2 \mathrm{~g} \mathrm{~cm}^{-3}$ \\
Atoms in the irradiated volume & $7.83 \times 10^{16}$ \\
Shear elastic modulus $\mu^{\mathrm{b}}$ & $63 \mathrm{GPa}$ \\
Reflectance $^{\mathrm{c}}$ & $\sim 0.18$ \\
Mean coordination number $\langle r\rangle$ & 2.4 \\
\hline
\end{tabular}

a Reference 12 .

${ }^{\mathrm{b}}$ Reference 50 .

${ }^{\mathrm{c}}$ Reference 51.

the values of $n$ obtained by fitting experimental photoviscosities with Eq. (10) are consistent with other photostructural effects. This, in turn, further reinforces the validity of Eq. (10) as a physically meaningful model for the mechanism of photoinduced fluidity.

Additionally, the proposed model was applied to the stoichiometric glass $\mathrm{As}_{2} \mathrm{~S}_{3}$ using published values of viscosity, absorption, and photodarkening ${ }^{12,19}$ (see Table VI). Figure 12 shows the experimental values of photofluidity obtained from Ref. 12 fitted with Eq. (10). The $\mathrm{As}_{2} \mathrm{~S}_{3}$ glass was irradiated with a He-Ne laser at a wavelength of $632.8 \mathrm{~nm}$ and the change in absorption due to photodarkening at this wavelength was estimated from Ref. 19 by assuming a linear variation between $a=1.55 \mathrm{~cm}^{-1}$ at $0 \mathrm{~W} / \mathrm{cm}^{2}$ and $a$ $=2.17 \mathrm{~cm}^{-1}$ at $100 \mathrm{~W} / \mathrm{cm}^{2}$. The shear modulus is $6.3 \mathrm{GPa}$ at room temperature and we suppose it becomes $15 \%$ lower under irradiation (any overestimation of the shear elastic modulus leads to an overestimation of $n$ with exactly the same proportion). The intensities used by Tanaka et al. ${ }^{12}$ for viscosity measurements range between 20 and $100 \mathrm{~W} / \mathrm{cm}^{2}$ so that the intensity absorbed (ranging between 0.6 and

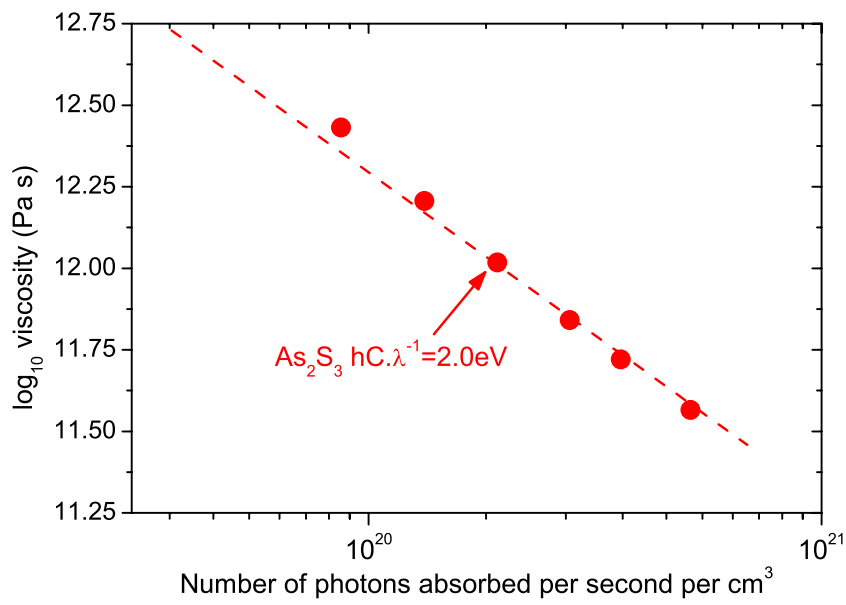

FIG. 12. (Color online) Evolution of the viscosity measured by Hisakuni and Tanaka (Refs. 9 and 12) as a function of number of photons absorbed per volume unit in $\mathrm{As}_{2} \mathrm{~S}_{3}$. The dashed line corresponds to our model.
4.2 $\left.\mathrm{W} / \mathrm{cm}^{2}\right)$ is in the same range than for the Ge-Se glasses studied here (this being illustrated by the density of photons absorbed indicated in Figs. 8 and 12). Figure 12 shows an excellent fit of the data for a factor $n=0.95$, which indicates a higher photon efficiency in $\mathrm{As}_{2} \mathrm{~S}_{3}$ than in Ge-Se glasses. This result is consistent with previous findings showing systematically larger photostructural changes in sulfides versus selenide glasses. ${ }^{52,53}$

It must be noted that the proposed model would appear to be in disagreement with previously reported photofluidity data in Ge-Se and As-S glasses. ${ }^{18,54}$ Gump et al. ${ }^{54}$ have concluded that a maximal photoinduced decrease in the elastic modulus $\left(C_{11}\right.$ or $\left.C_{L}\right)$ is observed at $\langle r\rangle=2.4$ in the Ge-Se system $\left(\mathrm{GeSe}_{4}\right)$. They suggest that this change results from a process mediated by the creation of self-trapped excitons. Our model directly correlating the viscosity to the efficiency of self-trapped excitons, we would expect that the composition corresponding to the lowest $\langle r\rangle$ would be the most sensitive to this change. Nonetheless, Gump et al. have measured $C_{11}$ in very large discrepancy (up to $60 \%$ larger) with any data in the literature. As an example, 26-21 GPa, as the intensity increases, for $\mathrm{Ge}_{15} \mathrm{Se}_{85}$ instead of $15.8 \mathrm{GPa}{ }^{55}$ Whatever the band-gap light intensities used by Gump et al. for irradiation, below at least $200 \mathrm{~W} \mathrm{~cm}^{-2}$, and whatever the composition, the $C_{11}$ measured are larger than expected values. So that it is not straightforward to conclude that a $C_{11}$ decrease exists in the Ge-Se system. Similarly, Yannopoulos et al. ${ }^{18,56}$ observed photofluidity in As-S fibers from the changes in Raman depolarization ratio resulting from structural reorientation in the direction of an applied stress. These measurements inferred a lower change for $\mathrm{As}_{1} \mathrm{~S}_{3}(\langle r\rangle=2.25)$ than for $\mathrm{As}_{2} \mathrm{~S}_{3}(\langle r\rangle=2.4)$. However a measure of structural orientation is a highly indirect way of quantifying the viscosity. Structural orientation in fibers may be affected by many factors, other than the viscosity, including preorientation due to the preparation method (drawing tension, etc.), ${ }^{57,58}$ the glass network dimensionality ${ }^{59}$ as well as thermal relaxation. ${ }^{60}$ Yannopoulos et al. did not conclude from the change in depolarization ratio that the viscosity is higher for the $\langle r\rangle=2.25$ composition. On the other hand, mean coordination sensitivities similar to the present study have been observed in Ge-Se glasses exposed to $\gamma$ irradiation: irradiation promotes smaller structural changes as the glass constraint increases. ${ }^{61}$ Our observations are also consistent with the conclusion of Wright et al. ${ }^{62}$ that, in the Ge-Se system, the photoinduced diffusion is rather the result of disturbances of Se-Se bonds than Ge-se ones.

\section{CONCLUSION}

It is shown that upon irradiation with wavelengths corresponding to sufficient penetration depths, photofluidity in chalcogenide glasses can be induced even under very low light intensity of $\sim 165 \mu \mathrm{W} / \mathrm{cm}^{2}$. These results indicate that no intensity threshold exists for photofluidity but instead suggest the existence of a simple relationship between light fluence and the induced fluidity. Proportionality appears between the time needed to induce a motion of all atoms within the irradiated volume and the viscosity. This proportionality 
holds over five orders of magnitude in irradiation intensity. Photoinduced fluidity can be modeled taking into account the glass absorption and its increase due to photodarkening. With a single parameter, both intensity and wavelength dependences can be modeled, from very low to high intensities, as long as the phenomenon stays athermal. This parameter $n$ can be seen as the photon efficiency: the number of atoms affected by a single photon absorption, in terms of atomic motions. The evolution of this parameter, determined by the study of photofluidity, shows a dependence on the mean coordination number similar to that of various photoinduced effects, such as photoexpansion, photoinduced structural relaxation, and photodarkening. It indicates that photoinduced effects are restricted by the number of constraints in the network: the greater the network constraints, the less atomic diffusions can occur. So that the photosensitivity is a monotonic function of the mean coordination number and is not sensitive to the well-known $\langle r\rangle=2.4$ threshold.

The model developed here agrees with the suggestion of Fritzsche,$^{5}$ that all the atoms of the glass network are affected by photoinduced effects. It shows that there is not a limited number of activated "knots" affected as suggested by
Tanaka. ${ }^{12}$ It then conforms to the idea that photoinduced effect are cumulative and that there is no reason to observe a threshold at low intensity to induce photofluidity. These results also have practical implication as they show that the role of even a weak light intensity cannot be neglected in the physical behavior of these chalcogenide glasses.

\section{ACKNOWLEDGMENTS}

The authors would like to thank Alain Moréac (Institute of Physics, University of Rennes 1, France), Ellyn A. King (Department of Materials Science and Engineering, University of Arizona), Gaëlle Delaizir (Equipe Verres et Céramiques, University of Rennes 1, France), Hervé Orain and Mickaël Le Fur (LARMAUR ERL CNRS 6274, University of Rennes 1, France) for experimental contributions, and Brice Arnaud (Institute of Physics, University of Rennes 1, France) for fruitful discussion. Y.G. thanks the French Ministry of Research (Grant No. 25094-2007). P.L. thanks NSFDMR under Grant No. 0806333 and NSF-ECCS under Grant No. 0901069 and the CNRS International Associated Laboratory for Materials \& Optics.
*Corresponding author; jean-christophe.sangleboeuf@univrennes1.fr

${ }^{1}$ M. Popescu, J. Optoelectron. Adv. Mater. 7, 2189 (2005).

${ }^{2} \mathrm{M}$. Popescu, Non-Crystalline Chalcogenides (Kluwer Academic, Dordrecht, 2000).

${ }^{3}$ R. Street, Solid State Commun. 24, 363 (1977).

${ }^{4}$ K. Tanaka, Solid State Commun. 34, 201 (1980).

${ }^{5}$ H. Fritzsche, Philos. Mag. B 68, 561 (1993).

${ }^{6}$ A. V. Kolobov, H. Oyanagi, K. Tanaka, and K. Tanaka, Phys. Rev. B 55, 726 (1997).

${ }^{7}$ M. Kastner, D. Adler, and H. Fritzsche, Phys. Rev. Lett. 37, 1504 (1976).

${ }^{8}$ O. U. Vonwiller, Nature (London) 104, 345 (1919).

${ }^{9}$ H. Hisakuni and K. Tanaka, Science 270, 974 (1995).

${ }^{10}$ M. Repka, M. Frumar, and M. Hrdlicka, J. Phys. Chem. Solids 68, 940 (2007).

${ }^{11}$ S. N. Yannopoulos and M. L. Trunov, Phys. Status Solidi B 246, 1773 (2009).

${ }^{12}$ K. Tanaka, C. R. Chim. 5, 805 (2002).

${ }^{13}$ B. V. Deryagin, Y. P. Toporov, K. I. Merzhanov, N. M. Gal'vids, I. N. Aleinikova, and L. N. Burta-Gapanavich, Sov. Phys. Solid State 16, 1155 (1974).

${ }^{14}$ A. Matsuda, H. Mizuno, T. Takayama, M. Saito, and M. Kikuchi, Appl. Phys. Lett. 25, 411 (1974).

${ }^{15}$ M. Trunov, J. Non-Cryst. Solids 192-193, 431 (1995).

${ }^{16}$ M. Trunov, V. Bilanich, and S. Dub, J. Non-Cryst. Solids 353, 1904 (2007).

${ }^{17}$ S. Nemilov and D. Tagantsev, Fiz. Khim. Stekla 7, 195 (1987).

${ }^{18}$ D. T. Kastrissios, G. N. Papatheodorou, and S. N. Yannopoulos, Phys. Rev. B 65, 165211 (2002).

${ }^{19}$ K. Tanaka, A. Saitoh, and N. Terakado, J. Optoelectron. Adv. Mater. 8, 2058 (2006).

${ }^{20}$ X. H. Zhang, Y. Guimond, and Y. Bellec, J. Non-Cryst. Solids
326-327, 519 (2003).

${ }^{21}$ J. Troles, V. Shiryaev, M. Churbanov, P. Houizot, L. Brilland, F. Desevedavy, F. Charpentier, T. Pain, G. Snopatin, and J. L. Adam, Opt. Mater. 32, 212 (2009).

${ }^{22}$ J. P. Guin, T. Rouxel, V. Keryvin, J.-C. Sangleboeuf, I. Serre, and J. Lucas, J. Non-Cryst. Solids 298, 260 (2002).

${ }^{23}$ C. Bernard, G. Delaizir, J.-C. Sangleboeuf, V. Keryvin, P. Lucas, B. Bureau, X.-H. Zhang, and T. Rouxel, J. Eur. Ceram. Soc. 27, 3253 (2007).

${ }^{24}$ P. Lucas, A. Doraiswamy, and E. A. King, J. Non-Cryst. Solids 332, 35 (2003).

${ }^{25}$ K. Fedus, G. Boudebs, C. B. de Araújo, M. Cathelinaud, F. Charpentier, and V. Nazabal, Appl. Phys. Lett. 94, 061122 (2009).

${ }^{26}$ L. Tichy, H. Ticha, P. Nagels, and R. Callaerts, J. Non-Cryst. Solids 240, 177 (1998).

${ }^{27}$ H. He and M. F. Thorpe, Phys. Rev. Lett. 54, 2107 (1985).

${ }^{28}$ Y. Gueguen, T. Rouxel, C. Bernard, J.-C. Sangleboeuf, V. Keryvin, and P. Gadaud (unpublished), high-temperature elasticity and viscosity of $\mathrm{Ge}_{x} \mathrm{Se}_{1-x}$ glasses in the transition range.

${ }^{29}$ G. Delaizir, J. Sangleboeuf, E. King, Y. Gueguen, X.-H. Zhang, C. Boussard-Pledel, B. Bureau, and P. Lucas, J. Phys. D: Appl. Phys. 42, 095405 (2009).

${ }^{30}$ I. Echeverría, P. L. Kolek, D. J. Plazek, and S. L. Simon, J. Non-Cryst. Solids 324, 242 (2003).

${ }^{31}$ Y. Gueguen, J.-C. Sangleboeuf, V. Keryvin, T. Rouxel, E. A. King, E. Robin, G. Delaizir, B. Bureau, X.-H. Zhang, and P. Lucas, J. Ceram. Soc. Jpn. 116, 890 (2008).

${ }^{32}$ R. Böhmer and C. A. Angell, Phys. Rev. B 48, 5857 (1993).

${ }^{33}$ J. DeBast and P. Gilard, Comptes rendus de recherche, vol. 1 (in French), Travaux du centre de technique et scientifique de l'industrie belge du verre, 1965.

${ }^{34}$ P. Lucas, E. A. King, A. Doraiswamy, and P. Jivaganont, Phys. Rev. B 71, 104207 (2005). 
${ }^{35}$ K. Tanaka, A. Saitoh, and N. Terakado, J. Mater. Sci.: Mater. Electron. 20, 38 (2009).

${ }^{36}$ E. H. Lee, Q. Appl. Math. 13, 183 (1965).

${ }^{37}$ P. Lucas and E. A. King, J. Appl. Phys. 100, 023502 (2006).

${ }^{38}$ C. R. Schardt, J. H. Simmons, P. Lucas, L. Le Neindre, and J. Lucas, J. Non-Cryst. Solids 274, 23 (2000).

${ }^{39}$ E. Lepine, Z. Zhiong, Y. Gueguen, J. Troles, X.-H. Zhang, B. Bureau, C. Boussard-Pledel, J.-C. Sangleboeuf, and P. Lucas, J. Opt. Soc. Am. B 27, 966 (2010).

${ }^{40}$ C. R. Schardt, P. Lucas, A. Doraiswamy, P. Jivaganont, and J. H. Simmons, J. Non-Cryst. Solids 351, 1653 (2005).

${ }^{41}$ H. Fritzsche, Solid State Commun. 99, 153 (1996).

${ }^{42}$ J. Li and D. A. Drabold, Phys. Rev. Lett. 85, 2785 (2000).

${ }^{43}$ G. A. N. Connell, Phys. Rev. B 24, 4560 (1981).

${ }^{44}$ G. Adam and J. Gibbs, J. Chem. Phys. 43, 139 (1965).

${ }^{45}$ K. Tanaka, Jpn. J. Appl. Phys. 25, 779 (1986).

${ }^{46}$ L. Calvez, Z. Yang, and P. Lucas, Phys. Rev. Lett. 101, 177402 (2008).

${ }^{47}$ M. Thorpe, J. Non-Cryst. Solids 57, 355 (1983).

${ }^{48} \mathrm{Z}$. El Gharras, A. Bourahla, and C. Vautier, J. Non-Cryst. Solids 155, 171 (1993).

${ }^{49}$ A. J. Moseley and J. M. Chamberlain, Philos. Mag. B 43, 1065 (1981).
${ }^{50}$ T. Rouxel, J. Am. Ceram. Soc. 90, 3019 (2007).

${ }^{51}$ G. Lucovsky, Phys. Rev. B 6, 1480 (1972).

${ }^{52}$ J. P. De Neufville, S. C. Moss, and S. R. Ovshinsky, J. NonCryst. Solids 13, 191 (1974).

${ }^{53}$ K. Tanaka, J. Non-Cryst. Solids 59-60, 925 (1983).

${ }^{54}$ J. Gump, I. Finkler, H. Xia, R. Sooryakumar, W. J. Bresser, and P. Boolchand, Phys. Rev. Lett. 92, 245501 (2004).

${ }^{55}$ S. S. Yun, H. Li, R. L. Cappelletti, R. N. Enzweiler, and P. Boolchand, Phys. Rev. B 39, 8702 (1989).

${ }^{56}$ D. Th. Kastrissios and S. N. Yannopoulos, J. Non-Cryst. Solids 299-302, 935 (2002).

${ }^{57}$ J. Murach and R. Brückner, J. Non-Cryst. Solids 204, 282 (1996).

${ }^{58}$ D. J. McEnroe and W. C. LaCourse, J. Am. Ceram. Soc. 72, 1491 (1989).

${ }^{59}$ H. Stockhorst and R. Brückner, J. Non-Cryst. Solids 85, 105 (1986).

${ }^{60}$ M. Ya, J. Deubener, and Y. Yue, J. Am. Ceram. Soc. 91, 745 (2008).

${ }^{61}$ R. Golovchak, A. Kozdras, S. Kozyukhin, and O. Shpotyuk, Nucl. Instrum. Methods Phys. Res. B 267, 2958 (2009).

${ }^{62}$ M. W. Wright and H. J. Trodahl, Phys. Rev. B 36, 4383 (1987). 